\title{
Development and implementation of flowing liquid lithium limiter control system for EAST
}

\author{
XiaoLin Yuan ${ }^{1,2}$, Yue Chen ${ }^{1}$, JianSheng $\mathrm{Hu}^{1 *}$, JianGang $\mathrm{Li}^{1}$, GuiZhong Zuo ${ }^{1}$, Jun Ren ${ }^{1}$, Yue Zhou ${ }^{1}$, \\ ChangZheng $\mathrm{Li}^{1}$,Zheng Sun ${ }^{1}$, Wei Xu ${ }^{1}$, XianCai Meng ${ }^{1}$, Ming Huang ${ }^{1}$, XingWei Zheng ${ }^{1}$, Xingjia \\ Yao ${ }^{1}$ and EAST team
}

1 Institute of Plasma Physics, Chinese Academy of Sciences, Hefei 230031, China

2 University of Science and Technology of China, Hefei 230031, China

\begin{abstract}
A control system of a flowing liquid lithium (FLiLi) limiter for the Experimental Advanced Superconducting Tokamak (EAST) was developed and implemented. The control system is not only able to control the direct current (DC) electromagnetic pump and heating power but can also set scanning parameters, receive the shot number, acquire the temperature, etc. The system consists of multifunctional LAN eXtensions for Instrumentation (LXI) instrument, temperature-acquisition module, programmable DC power supply, and programmable logic controller (PLC). The multi-range DC power supply is programmed to meet the operational requirements of the DC electromagnetic pump. The LXI instrument and temperature-acquisition module are used to obtain temperature data. The PLC is adopted to control the temperature of the FLiLi limiter. A safety interlock and protection function was developed for the FLiLi limiter control system. The software was designed by using LabVIEW to achieve data interaction between multiple protocols. The FLiLi limiter control system can acquire experimental data at a speed of $100 \mathrm{~S} / \mathrm{s}$ and store it for later analysis. The control system was successfully applied to a FLiLi limiter to study the interaction between plasma and a fixed wall in the EAST campaign. This paper presents the framework, the implementation details, and results of the control system.
\end{abstract}

Keywords: EAST, Flowing liquid lithium limiter, LabVIEW, LXI

\section{Introduction}

The Experimental Advanced Superconducting Tokamak (EAST) is the first fully superconducting tokamak with a D-shaped cross-section for plasma in the world. The scientific mission of the EAST project is to study the physics involved in advanced steady-state tokamak devices [1-3]. With the improvement of EAST discharging parameters, traditional solid plasma-facing components (PFCs) now present a significant challenge. Liquid Li has low ionization energy as a PFC and can be easily ionized at the edge of plasma [4-6]. The successful implementation of a flowing liquid lithium (FLiLi) limiter control system is crucial to FLiLi operation for studying plasma-facing material in fusion research devices [7-9].

The control system for the EAST FLiLi limiter can remotely control the drive current of the DC electromagnetic (EM) pump, Li heating system, intelligent device network, and temperature recording system. The real-time temperature and intelligent device state can also be monitored. An LXI instrument with a temperature-acquisition module was adopted to realize high-speed data acquisition and transfer in a local area network (LAN) [10]. Intelligent DC power was adopted to detect the power self-state and show the parameters on the operation interface [11-13]. This design method improves the reliability of the system. To protect the safety of the FLiLi limiter, a safety interlock and protection function was designed. The human-machine interface (HMI) was designed by using the $\mathrm{G}$ language (LabVIEW). The G language is not only helpful for faster program development but also for realizing an interface with good human-computer interaction $[14,15]$.

*Corresponding author e-mail: hujs@ipp.ac.cn 


\section{System description}

The main part of the FLiLi limiter is composed of a copper plate with a length of $350 \mathrm{~mm}$ and width of 300 $\mathrm{mm}$. A thin $(0.1 \mathrm{~mm})$ stainless steel (SS) foil brazed to the copper plate serves as a guide plate and protective layer between the liquid Li and copper heat sink.

Fig. 1 shows a schematic diagram of the EAST FLiLi limiter. The innovative distributor on top of the limiter consists of 200 small $(0.8 \times 0.8 \times 40 \mathrm{~mm} 3)$ channels so that the liquid $\mathrm{Li}$ flows down the plasma-facing surface of the guide plate uniformly. The bottom collector, which is separated from the bottom of the plasma-facing side of the guide plate by a rectangular gap, is designed to store liquid Li. Two electrodes attached to the bottom of the FLiLi limiter work as a DC EM pump that provides a current crossing the section of the injection Li pipe. With the strong toroidal field of EAST Bt, the liquid $\mathrm{Li}$ is driven by the $J \times B$ force.

As shown in Fig. 1, the system uses the DC EM pump to inject liquid $\mathrm{Li}$ into the distributor on top of the limiter via the injection Li pipe. The injection pressure can be controlled by the driver current of the EM pump. Then, the viscous liquid Li slowly flows $(<10 \mathrm{~mm} / \mathrm{s})$ out from the distributor to the bottom collector along the guide plate of the limiter due to gravity. The structure realizes a liquid $\mathrm{Li}$ loop along the top distributor and the front surfaces of the limiter and bottom collector. To keep the liquid Li moving on the surface, the limiter needs to be maintained at an appropriate temperature by heater strips installed in the heater groove. To exhaust the heat load from the burning plasma effectively and protect the limiter surface from an unexpected rise in temperature, the cooling channels are installed on the back side of the guide plate. The cooling system consists of channels that circulate $\mathrm{He}$ gas at $5 \mathrm{MPa}$.

A programmable logic controller (PLC) was adopted to control the power of the heater strips and adjust the limiter temperature. The LXI instrument with the temperature-acquisition module was applied to realize high-speed data acquisition for later analysis.

The working conditions of the FLiLi control system were more complex especially during the EAST campaign. In this case, the FLiLi control system required reliable and stable hardware and robust software to ensure successful application.

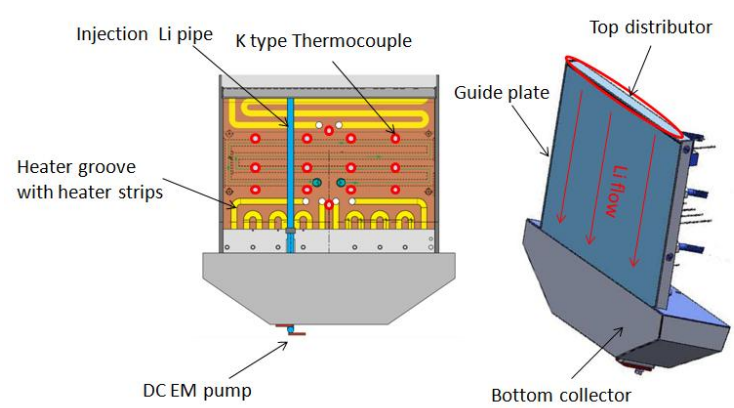

Fig. 1. Schematic diagram of flowing liquid lithium limiter.

\subsection{Hardware system design}

\subsubsection{Hardware architecture}

Intelligent instruments were chosen to meet the reliability and stability requirements of the FLiLi limiter control system. The hardware control architecture has three layers, as shown in Fig. 2: the device layer, controller layer, and operator interface (OPI) layer.

The device layer consists of 14 K-type thermocouples, a DC EM pump, and three heating powers, each of which can reach a power output of 2.5 $\mathrm{kW}$.

Before plasma operation is initiated with the FLiLi limiter, it is necessary to use heating strips to preheat the limiter and wet the surface with liquid Li. The heating strips are fixed in heater grooves and are insulated with an alumina coating. The thermocouples are used to detect the temperature of the heat sink from which it can be evaluated the temperature of the limiter surface. The DC EM pump was designed to supply a continuous flow of liquid Li to the limiter, and the driving pressure provided to the liquid Li can be controlled by changing the pump's parameters.

The controller layer consists of an LXI instrument with a temperature-acquisition module, programmable DC power supply, and PLC module. For this control system, an LXI instrument (Agilent 34972A) with a temperature-acquisition module was adopted to acquire the temperature data. In this framework, the LXI instrument is an Ethernet network node that is controlled by an industrial personal computer (IPC). This setup is widely used for distributed applications and remote control. To achieve reliable control of the DC EM pump, a programmable DC power supply was chosen. By setting the current or voltage slew rate, the DC power supply can work in current or voltage mode. Those parameters can be set on the OPI. The Siemens PLC (CPU 315-2 DP) with an analog output module was used to adjust the heating power of the heater strips in the grooves.

All instruments are connected to a LAN router with a static IP address that is assigned manually. An IPC was 
adopted as a master station to communicate with all instruments. The operators send commands to and configure information for instruments with the IPC. All measured data are transmitted to the IPC for the monitoring and storage.

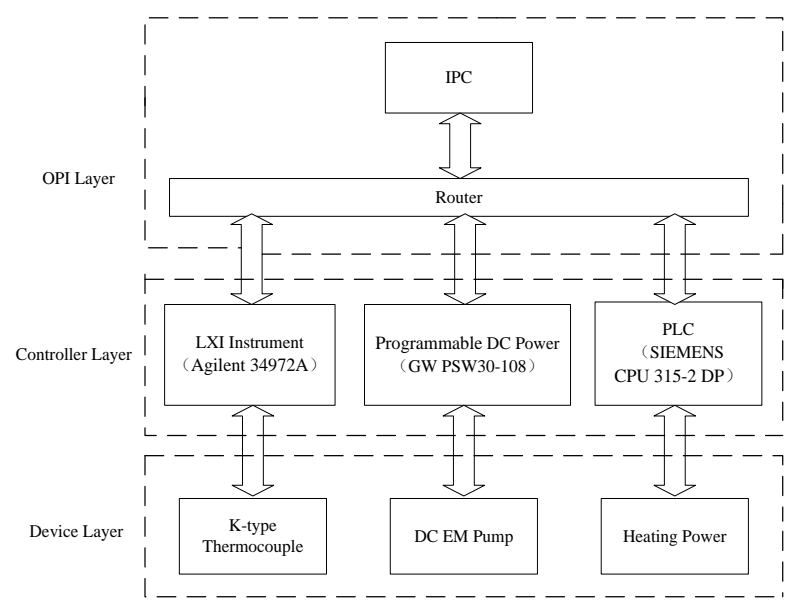

Fig. 2. Architecture of the hardware system.

\subsubsection{Safety interlock and protection}

Performing a FLiLi limiter experiment under poor vacuum conditions is very dangerous. For example, Li sharply reacts with water when a faulty vacuum causes air or water to leak from the heat sink coolant. To avoid such damage, a safety interlock and protection function was developed for the FLiLi control system to resolve the running risks.

As shown in Fig. 3, the EAST vacuum control system (VCS) sends a hardware trigger signal to the FLiLi limiter control system if the plasma vacuum vessel (PVV) pressure is more than $1000 \mathrm{~Pa}$. When it receives the trigger, the FLiLi limiter control system starts a protective function: the FLiLi control system stops heating of the limiter, while Ar gas is injected in the tokamak device.

A special liquid level detection device is used to detect the flowing liquid level in the collector. The FLiLi limiter control system sends an error signal to the EAST VCS if the flowing liquid is over the safety range. At the same time, the FLiLi limiter stops the limiter heating, and starts the cooling system. If the FLiLi limiter passes self-detection, the FLiLi limiter control system sends a ready signal to the EAST vacuum control system.

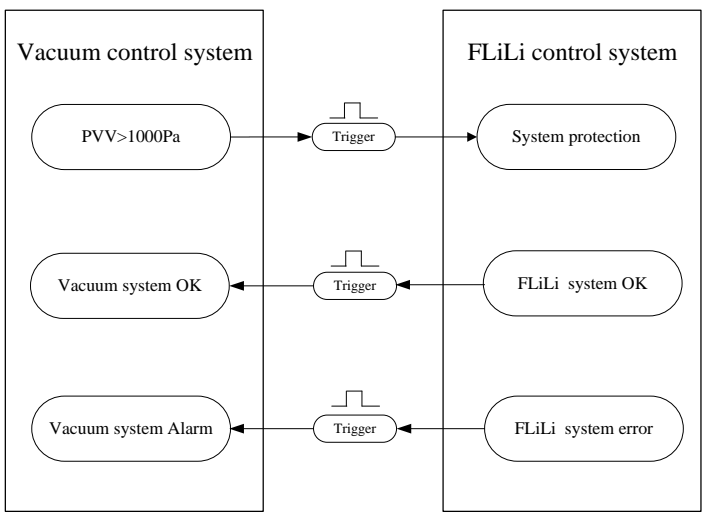

Fig. 3. Safeguard between vacuum control system and FLiLi control system

\subsection{Software system design}

\subsubsection{Software architecture}

To realize interaction between the data of different instruments, a multiprotocol data transmission software was designed that contains Transmission Control Protocol (TCP), User Datagram Protocol (UDP), and the Profinet communication protocol. Fig. 4 shows the software architecture of the overall control system.

The PLC is connected to an OLE for Process Control (OPC) server that is embedded in the LabVIEW platform by the Profinet communication protocol. As part of the EAST system, it needs to be synchronized with the plasma control system (PCS). UDP is used to connect to the PCS in order to receive a discharge message. In the UDP message, the shot number and discharge time span are separated with a comma.

The multiprotocol platform can access the programmable DC power supply and LXI instrument via TCP/IP. A software filter was adopted to discard singular values and help to obtain a precise limiter temperature. 


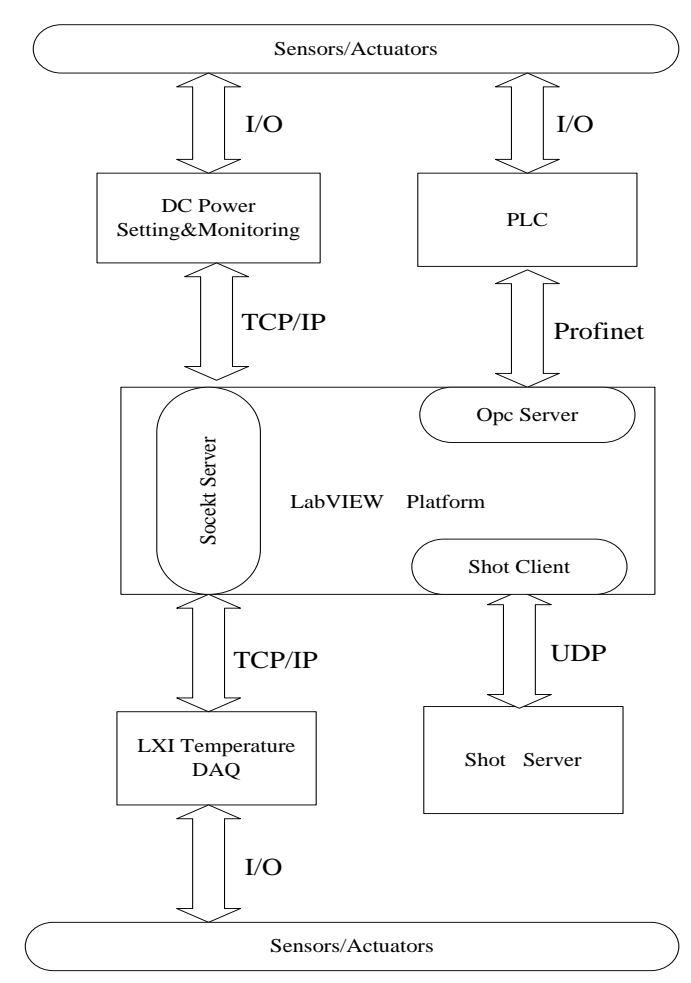

Fig. 4. Architecture of the Software system.

\subsubsection{Workflow of software}

Fig. 5 shows the workflow of the control system. When the system starts, the OPC is initialized to build a connection between the PLC and LabVIEW control platform. Then, the LXI instrument starts self-detection if the scanning frequency, thermocouple type, trigger mode, and IP address are successfully configured. The programmable DC power supply checks the DC EM pump setting parameter to see if it satisfies the protective values.

If these conditions meet the requirements, the control system sends a ready signal to the VCS and starts to acquire temperature data and receive discharge information. The limiter temperature data during discharge and discharge messages are saved for later physical analysis. The system protective function is started if there is a FLiLi limiter error or PVV of more than $1000 \mathrm{~Pa}$. To protect the heater strips, heating powers are halted during the discharge. After discharge, the heating powers resume. The multiprotocol platform was programmed with LabVIEW. This graphical programming language is useful not only for visual programming but also for easy maintenance. Fig. 6 shows the OPI of the control system.

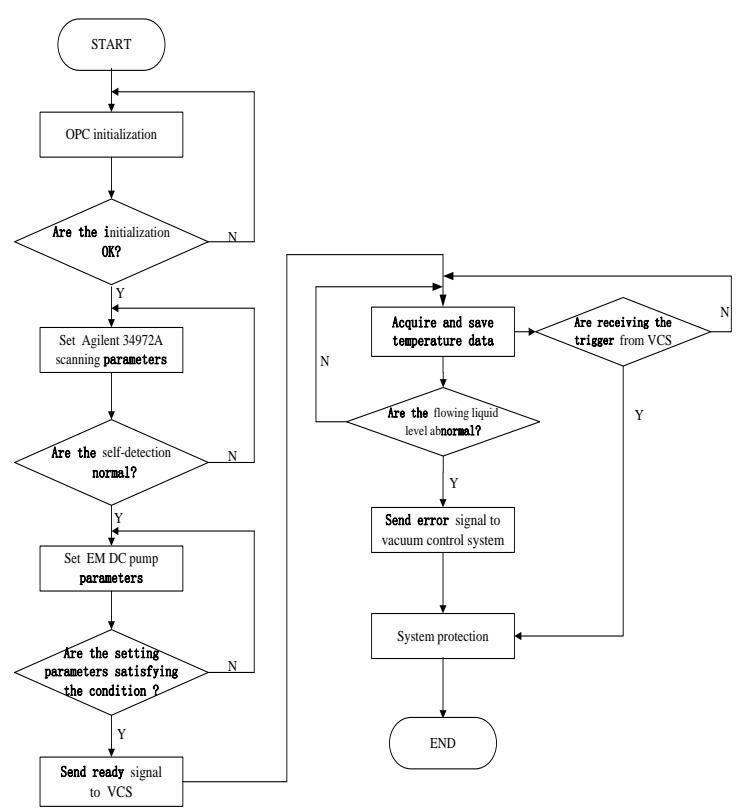

Fig. 5. Workflow of system.

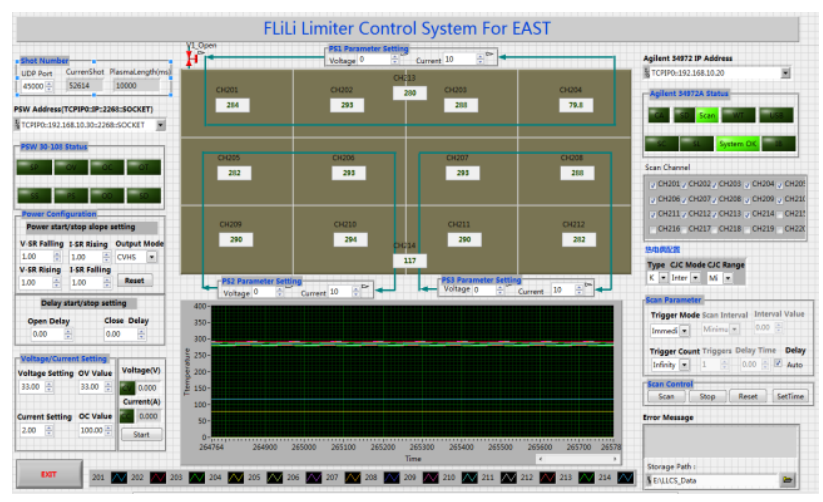

Fig. 6. Operator Interface of control system

\section{Results}

Fig. 7(a) shows the installation of the FLiLi limiter. Fig. 7(b) shows the FLiLi control system hardware.

Fig. 8 shows the FLiLi experiments on EAST, which were influenced by applying different driving currents to the DC EM pump. The light area on the limiter indicates strong interaction between the FLiLi and plasma. The plasma performance was improved by using FLiLi under both the L-mode and H-mode plasma conditions [16]. The programmable DC power output produced a different driving current in the DC EM pump to drive the Li flow on the limiter surface. Thus, the controllability of the DC EM pump was verified in the experiment.

According to the Nyquist sample theorem[17], the sampling rate must be more than twice the maximum frequency of the device signal being measured. However, a sampling rate of ten times the maximum frequency of the device signal is usually adopted in practical engineering. The maximum frequency of the system thermocouple was $10 \mathrm{~Hz}$, so the control system sampling rate was set to $100 \mathrm{~S} / \mathrm{s}$. Fig. 9 shows the temperature 
curves of the same measurement point with different driving currents.

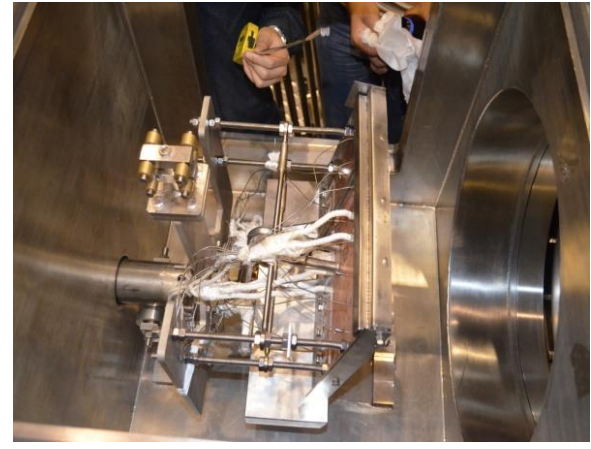

(a) Flowing liquid lithium limiter

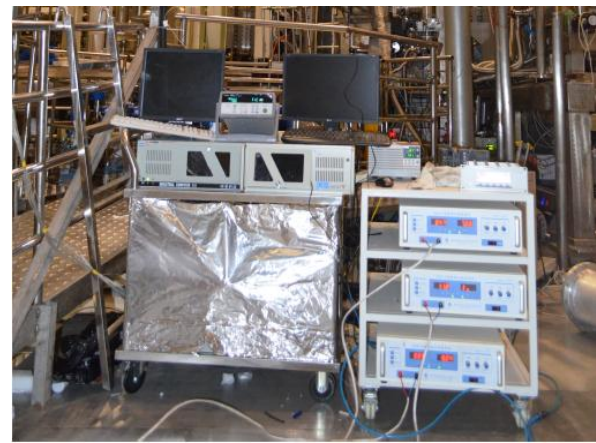

(b) FLiLi control system hardware

Fig. 7. A picture of the FLiLi experiment on EAST.

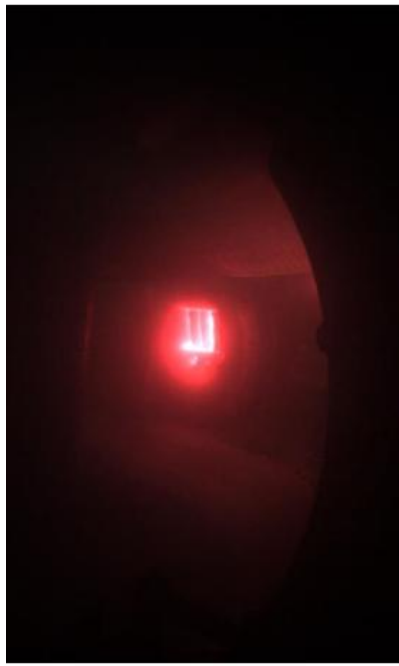

(a) $40 \mathrm{~A}$

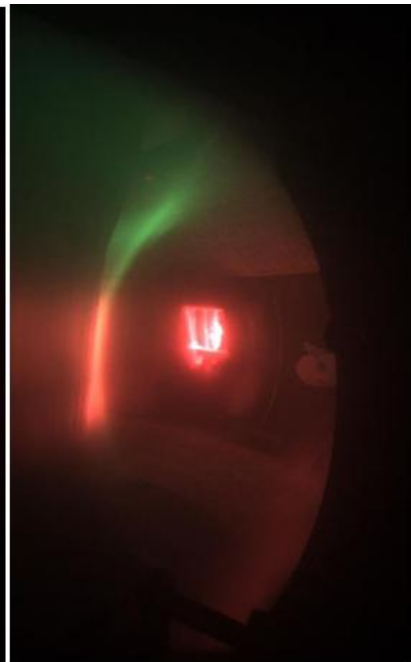

(b) $80 \mathrm{~A}$
Fig. 8. The phenomenon of different driving current on the EM pump

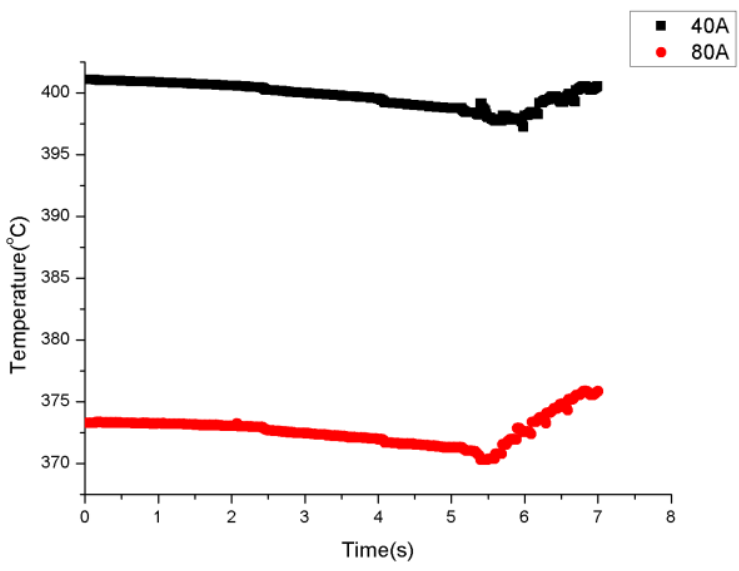

Fig. 9. The temperature curve of same measuring point with different driving current

\section{Conclusion}

The FLiLi control system hardware consists of an LXI instrument with a temperature-acquisition module, a programmable DC power supply, and a PLC module. The software employs a graphical programming language to simplify the programming process. The FLiLi control system was subjected to an experiment on a test platform to determine its capabilities. The test results showed that the system can control the drive capability of the DC EM pump, acquire the limiter temperature, control the heating power, and manage the equipment reliability.

The system was successfully applied to the 2014 EAST campaign. The FLiLi control system is essential to the operation of EAST. FLiLi has helped the research on the interaction between plasma and a liquid metal as first wall material. It will be upgraded to meet new requirements of a future EAST campaign.

\section{Acknowledgments}

This research is funded by National Magnetic confinement Fusion Science Program under Contract No. 2013GB114004 and National Nature Science Foundation of China under Contract No. 11321092 and No. 11405210. This work was also partly supported by the Japan Society for the Promotion of Science-National Research Foundation of Korea-National Science Foundation of China (JSPS-NRF-NSFC) A3 Foresight Program in the field of Plasma Physics (NSFC No. 11261140328).

\section{References}

[1] S.V. Mirnov, A.M. Belov, N.T. Djigailo, et al. Nucl. Fusion., 55(2015) 123015

[2] J. Li, H. Y. Guo, B. N. Wan, et al. Nat. Phys., 9(2013)817 
[3] J. S. Hu, Z. Sun, H. Y. Guo, et al. Phys. Rev. Lett., 115(2015) 169901

[4] Igor E. Lyublinski, Alexey V. Vertkov. Fusion Eng. Des., 85(2010) 924-929.

[5] A. Bertocchi, M. Di Donna, M. Panella, V. Vitale. Fusion Eng. Des., 82(2007) 961-965.

[6] M.L. Apicella, V. Lazarev, I. Lyublinski, G. Mazzitelli, S. Mirnov, A. Vertkov, et al. J. Nucl. Mater., 386-388 (2009) 821-823

[7] R. Majeski, S. Jardin, R. Kaita, T. Gray, P. Marfuta, J. Spaleta, et al. Nucl. Fusion., 45 (2005) 519-523.

[8] J.Ren, G.Z.Zuo, J.S.Hu, Z.Sun, Q.X.Yang, J.G.Li, et al. Rev. Sci. Instrum., 86 (2015) 023504

[9] M. Narula, M.A. Abdou, A. Ying, N.B. Morley, M. Ni, R. Miraghaie, et al. Fusion Eng. Des. 81 (2006)1543-1548.

[10] Liu Zhao-qing, Pan Shao-wu, Zhang Yi-gang. Measurement. 46 (2013) 2753-2764

[11]Joby Antony, D. S. Mathuria, et al. Rev. Sci. Instrum. 86(2015) 125003

[12] Min Li, Y.J. Yuan, R.S. Mao, et al. Nuclear Instruments and Methods in Physics Research A, 776 (2015) 21-26

[13] Simone Mannori, Valerio Sermenghi, et al. Fusion Eng. Des., 88(2013) 687-691.

[14] Chengquan Pei, Shengli Wu, Jinshou Tian, Zhen Liu, et al. Nuclear Instruments and Methods in Physics Research A, 799(2015)124

[15] J. Jugo, M. Eguiraun, I. Badillo, et al. IEEE Transactions on Nuclear Science, 62 (2015) 889-896.

[16] J. S. Hu, G.Z. Zuo, J.Ren, et al. Nuclear Fusion., 56(2016)046011.

[17] Xiangdong Huang, Yiwen Han, et al. IEEE Transactions on Signal Processing, 64 (2016) 2489-2498. 